\title{
CORRESPONDENCE
}

\section{KNICK POINTS AND PROFILES}

Sir,- In his most valuable account of Wealden geography, Professor Allen uses the objectionable term nick points (Geol. Mag., xci, 500). At none of these points is there any nick or nitch or notch, such as an undercut waterfall; only a flaw in the curve, like the broken back of a beast.

The term originated in the German Knickpunkt. It is true that knick does not appear in modern English dictionaries, though it lingers disguised in the knacker's yard. But that is surely no reason why a word with a totally different meaning should be used instead, merely because it has the same sound. It is as though an innkeeper should say he had no beer but there was a perfectly good bier available.

Another point - why do some geographers talk of the long profile and the cross profile of a valley? In common language a profile is a side view. At the Royal Academy we may see portraits in profile, half profile, or full face, smiling, smug perhaps, but never cross. What is wrong with profile and cross-section in the case of a valley?

G. M. Davies.

63 BEECHWOOD ROAD,

SANDERSTEAD, SURREY.

13th December, 1954.

\section{HORIZONTAL STRESS AND TRANSCURRENT FAULTS}

StR,-In reply to H. W. Wellman's letter (Geol. Mag., xci, 1954, 407-8) on the angles between principal horizontal stresses and transcurrent faults, I would like to make the following comments.

The writer refers in the first place to Leedal and Walker's investigation, and map (p. 118) of the Lough Belshade and Barnes Lough Faults in Northern Ireland. He says that, in virtue of a principle which I have advocated, " and without confirmatory evidence," Leedal and Walker have concluded that the two faults must be of different ages. On pp. 119 and 120 of their paper the two authors do, however, give some confirmatory evidence, although they do not claim that it is conclusive.

The writer next cites the work of J. B. Auden in Gujarat (India), and the map presented by him on p. 94 of the same volume. Auden, however, in referring to the supposed transcurrent faults involved, says that "in Gujarat the fracturing is mainly vertical ", and this leaves one very much in doubt whether he may not be dealing with two non-contemporaneous systems of normal faulting.

There is further reference to "two suitable pairs of active transcurrent faults" in New Zealand, investigated by the writer himself. In the map which accompanies the cited paper it is easy to trace the "Alpine Fault", and identify the "Moonlight Fault", but no indication appears to be given, either in the map or the list of dislocations, with regard to the other pair. Unless Mr. Wellman has published elsewhere, the information available appears to be far too fragmentary for the formation of any conclusions.

The last case cited is that of the San Andreas Fault, along with the supposed complementary Big Pine and Garlock Faults. These intersect at an angle much greater than $90^{\circ}$, as may be gathered from the writer's statements. Both the San Andreas Fault, with its parallels, and the other two mentioned are active at present: I have long known and been puzzled about this discrepancy, but perhaps the explanation is as follows:-

As is well known, when there are three principal stresses in any medium, of different magnitudes, any fracture which may result must be parallel, or nearly so, to the one which is intermediate in value. The inclination of the

voL. XCI.-NO. 1. 